\title{
Sonography of the Bladder After Ureteral Reimplantation
}

\author{
J. Michael Zerin, MD*, Jeffrey D. Smith, DO' ${ }^{\dagger \ddagger}$, Jill K. Sanvordenker, RN ${ }^{\dagger}$, David A. Bloom, MD
}

Pre- and postoperative sonograms were reviewed in 59 children who underwent ureteral reimplantation to assess the frequency, location, extent, and evolution over time of focal thickening of the posterior bladder wall and bladder base after reimplantation. The interval between reimplantation and follow-up sonography ranged from 2 weeks to 12.36 years (mean, 1.29 years).

Thirty-two patients $(54.2 \%)$ had focal thickening of the posterior bladder wall and trigone after reimplantation. The earlier after reimplantation the children were first evaluated with sonography, the much more frequently was thickening observed. Thirteen had fusiform thickening along the submucosal tunnel and twenty-four had a hyperechoic nodule at the trigone. The thickening resolved in one third of the children and is presumed to have represented postoperative edema in these cases. However, on the sonographic appearance alone it was not possible to differentiate transient changes resulting from postoperative edema from those representing developing fibrosis or granuloma formation. KEY wORDS: Bladder, Ultrasound, Ureteroneocystostomy.
$\mathrm{U}$ reteroneocystostomy (ureteral reimplantation) is one of the most commonly performed uroIogic surgical procedures in children. ${ }^{1,2}$ Vesicoureteral reflux (VUR) and ureterovesical junction (UVJ) obstruction are the most common indications for reimplantation of the ureter. Reimplantation may also be required in the course of resection of a ureterocele or bladder diverticulum.

The cross-trigonal ureteral advancement procedure (Cohen reimplantation) ${ }^{3,4}$ (Fig. 1A) is the most common technique employed in our hospital, whereas the Leadbetter-Politano technique (LP reimplantation) ${ }^{5}$ (Fig. 1B) is performed less frequently. The course and length of the submucosal ureteral tunnel, as well as the location of the ureteral orifice within the bladder, are altered in both procedures. The resulting changes in the appearances of the distal ureter and bladder on urography and cystography have been described previously after

Received March 14, 1991, from the Sections of "Pediatric Radiology and Urology, Department of Surgery, University of Michigan Hospitals, Ann Arbor, Michigan. 'Present address: Department of Radiology, Botsford General Hospital, Farmington Hills, Michigan. Revised manuscript accepted for publication July 23, 1991.

Address correspondence and reprint requests to Dr. J. Michael Zerin: Section of Pediatric Radiology, University of Michigan Hospitals, 200 E. Hospital Drive, Ann Arbor, MI 48109-0252. both types of reimplantation and are well known.' Sonographically visible changes in the course of the submucosal ureter (tunnel sign) and thickening of the bladder base and posterior bladder wall have been noted after cross-trigonal reimplantation, ${ }^{6}$ although the spectrum of appearances of these changes in the bladder wall and their evolution over time have not, to our knowledge, been investigated previously.

\section{MATERIALS AND METHODS}

Review of records in the pediatric radiology and urology departments led to the identification of 87 children who had undergone ureteral reimplantation (or who had been referred with a history of reimplantation) and who had had one or more urinary tract sonographic examinations performed during the past 3 years. Twenty-five patients in whom images of the full bladder were not available on any studies done after reimplantation and three patients who had other bladder surgery in addition to reimplantation (two had vesicostomy and one had bladder augmentation) were excluded. The medical records, surgical reports, and sonograms were reviewed in the remaining 59 patients (15 male and 44 female patients). 


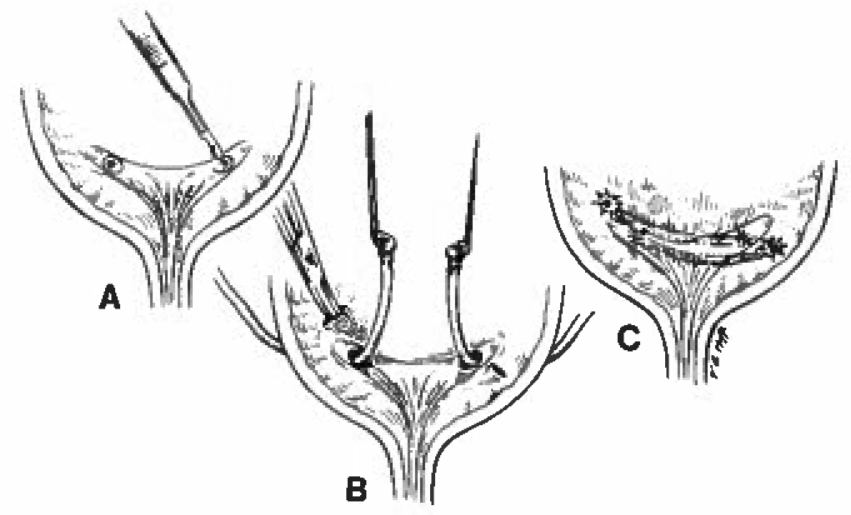

$\mathbf{A}$

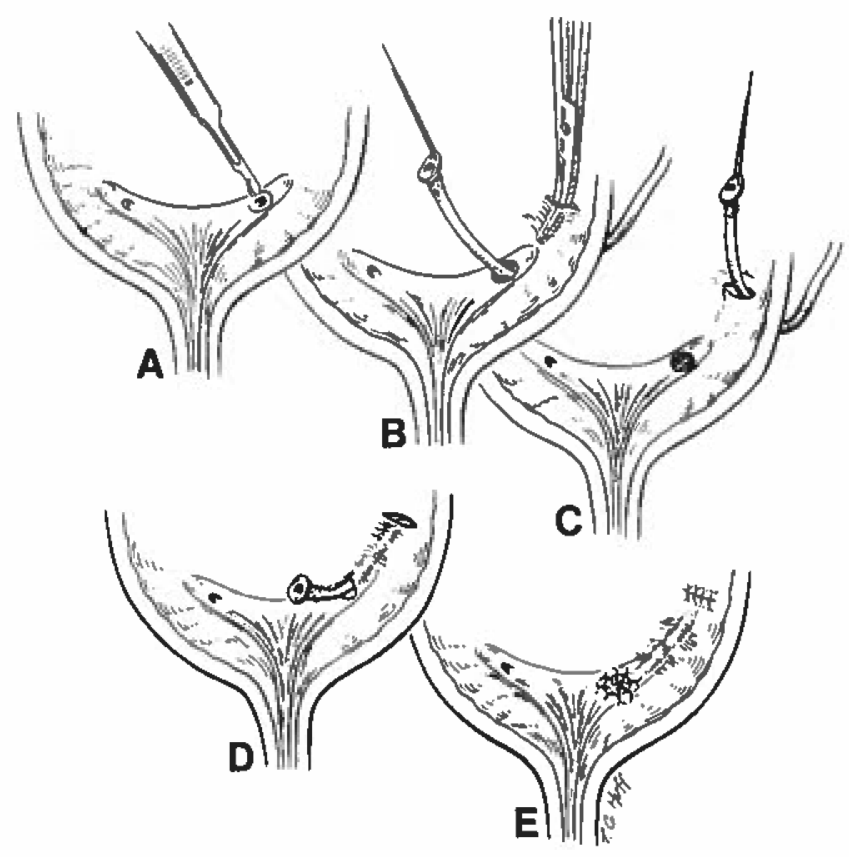

B

Figure 1 A, Cross-trigonal (Cohen) procedure for reimplantation of the ureter. Incisions are made around the internal ureteral orifice, freeing the ureter from its attachments on the trigone. The ureter is then advanced across the trigone to the contralateral side, lengthening the submucosal tunnel, and a new internal ureteral orifice is created. B, Leadbetter-Politano procedure for reimplantation of the ureter. Incisions are made around the internal ureteral orifice, freeing the ureter from its attachments on the trigone. The ureter is passed outside the bladder through the bladder wall incision. A higher and more medial opening is made in the bladder wall through which the ureter is brought back into the bladder wall and extended by blunt dissection toward the bladder neck, where a new internal ureteral orifice is created.

Fifty-seven patients $(96.6 \%)$ underwent cross-trigonal reimplantation and two patients $(3.4 \%)$ underwent LP reimplantation. Twenty-six reimplantations were bilateral. Two patients who had undergone bilateral LP reimplantation previously elsewhere for bilateral VUR required unilateral re-reimplantation (cross- trigonal), in one case for persisting unilateral reflux and in the other for unilateral UVJ obstruction. The indications for reimplantation were VUR in 52 patients (88.1\%), UVJ obstruction in 7 patients $(11.9 \%$ ) (primary megaureter in six and postsurgical obstruction in one), ureterocele in four patients $(6.6 \%)$, and paraureteral bladder diverticulum in one patient (1.6\%). Eight patients had reimplantation of upper and lower pole ureters on the side of a duplicated system. Multiple indications for reimplantation were present in five patients.

Both pre- and post-reimplantation sonograms were reviewed in each patient. The bladder was sufficiently distended to be evaluated on pre-reimplantation sonography in 54 patients. The bladder was adequately distended on 115 post-reimplantation sonograms in the 59 patients. Thirty-two patients had multiple postreimplantation studies (range, two to five studies per patient). All of the sonographic examinations were sector scans done on either an Acuson 128 or a GE RT3600 machine with either $3 \mathrm{MHz}$ or $5 \mathrm{MHz}$ transducers. Because of the retrospective nature of this investigation only the "hard copy" images could be reviewed. The films were evaluated jointly by two radiologists (JMZ, JDS), and assessments of the presence of focal bladder wall thickening were based on the consensus of these two observers. When present, the location and extent of focal thickening of the posterior bladder wall and bladder base (FBWT) were noted, and the thickening was categorized as either "fusiform" or "nodular." Broad-based thickening of one or both sides of the trigone along the expected course of the reimplanted submucosal ureter was designated "fusiform," whereas thickening producing the appearance of a sessile or pedunculated mound of tissue projecting into the bladder lumen was designated "nodular." The two patterns of thickening coexisted in some patients.

\section{RESULTS}

At the time of operation, the patients ranged in age from 1 month to 17,0 years (mean, 5.81 years). The mean interval between reimplantation and the first post-reimplantation sonographic study was 1.29 years (range, 12 days to 12.36 years). The mean duration of follow-up post-reimplantation was 2.07 years (range, 12 days to 12.64 years).

Thirty $(52.6 \%)$ of the 57 patients who underwent cross-trigonal reimplantation and both of the patients who underwent LP reimplantation had FBWT on postreimplantation sonography that had not been present on preoperative studies. Correlation of postoperative sonographic findings with the urologic diagnosis prior to reimplantation is summarized in Table 1. FBWT was 
Table 1: Findings on Post-reimplantation Sonograms in Relation to Urologic Diagnosis Prior to Reimplantation

\begin{tabular}{lcc}
\hline \multirow{1}{*}{$\begin{array}{c}\text { Indication for } \\
\text { Reimplantation }\end{array}$} & \multicolumn{2}{c}{$\begin{array}{c}\text { Post-reimplantation } \\
\text { Sonogram }\end{array}$} \\
\cline { 2 - 3 } & No FBWT & FBWT \\
\hline Primary reflux only $(\mathrm{n}=43)$ & $26(49.0 \%)$ & $17(60.5 \%)$ \\
UVJ obstruction† $(\mathrm{n}=7)$ & $1(14.3 \%)$ & $6(85.7 \%)$ \\
Ureterocele† $(\mathrm{n}=4)$ & $0(0 \%)$ & $4(100 \%)$ \\
Diverticulum $(\mathrm{n}=1)$ & $0(0 \%)$ & $1(100 \%)$ \\
Duplex system $(\mathrm{n}=8)$ & $0(0 \%)$ & $8(100 \%)$ \\
\hline
\end{tabular}

- Single system ureter.

t With or without reflux.

to reimplantation is summarized in Table 1. FBWT was more frequent in patients in whom the reimplantation involved excision of a ureterocele or bladder diverticulum or repair of UVJ obstruction, as opposed to those with VUR. However, the number of children with preoperative urologic diagnoses other then VUR is too small to evaluate the statistical significance of these differences.

The earlier after reimplantation that the children were first evaluated with sonography, the more frequently was FBWT observed. The relationship between the time of the first follow-up sonographic examination and the presence of FBWT is summarized in Table 2. FBWT was significantly more frequent in the first 6 months after operation than after this period. Thickening was seen in four ( $80 \%$ ) of five children studied within the first month after reimplantation and in eight (66.7\%) of 12 children first studied in the second month after reimplantation, but it was seen in only seven (33.3\%) of 21 patients first studied more than 6 months after reimplantation. There was no relationship between patient sex or age at operation and the development of FBWT after reimplantation.

Thirteen patients had fusiform thickening of the posterior bladder wall and trigone along the expected course of the submucosal tunnel of the reimplanted ureter (Fig. 2). This thickening was bilateral in seven patients. The thickening decreased in severity on fol-

Table 2: Findings on Post-reimplantation Sonograms in Relation to the Timing of the First Sonographic Study after Operation

\begin{tabular}{clrr}
\hline \multirow{2}{*}{$\begin{array}{c}\text { Days after } \\
\text { Reimplantation }\end{array}$} & \multicolumn{2}{c}{$\begin{array}{c}\text { Post-reimplantation } \\
\text { Sonography }\end{array}$} \\
\cline { 3 - 4 } & & \multicolumn{1}{c}{ No FBWT } & \multicolumn{1}{c}{ FBWT } \\
\hline $0-30$ & $(\mathrm{n}=5)$ & $1(20.0 \%)$ & $4(80.0 \%)$ \\
$31-60$ & $(\mathrm{n}=12)$ & $4(33.3 \%)$ & $8(66.7 \%)$ \\
$61-180$ & $(\mathrm{n}=21)$ & $8(38.1 \%)$ & $13(61.9 \%)$ \\
$181-365$ & $(\mathrm{n}=7)$ & $5(71.4 \%)$ & $2(28.6 \%)$ \\
$>365$ & $(n=14)$ & $9(64.3 \%)$ & $5(35.7 \%)$ \\
All patients & $(\mathrm{n}=59)$ & $27(45.8 \%)$ & $32(54.2 \%)$ \\
\hline
\end{tabular}

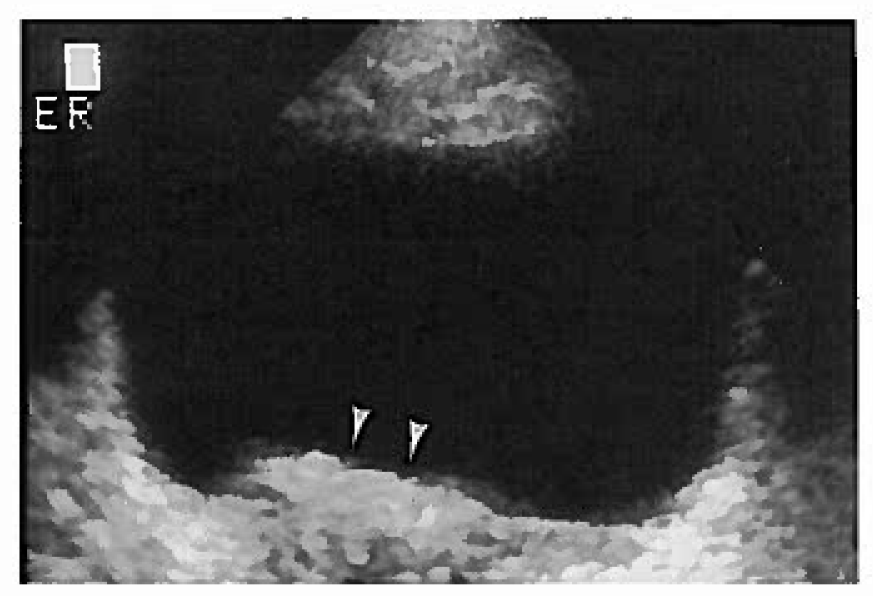

Figure 2 Transverse sonogram of the bladder 4 months after uncomplicated bilateral cross-trigonal reimplantation for bilateral lower pole reflux in a 16 month old girl. Fusiform thickening of the bladder base along the expected course of the reimplanted submucosal ureters is noted (arrowheads).

low-up examinations in seven of 11 patients who had multiple post-reimplantation sonograms and disappeared entirely in four patients.

Twenty-four patients $(40.7 \%)$ had an echogenic mural nodule visible on the trigone, which projected into the lumen of the bladder. The nodules were either sessile (Fig. 3) or pedunculated (Fig. 4) and ranged in diameter from several millimeters to several centimeters. Although precise evaluation of changes in the size of the nodules on successive examinations was difficult in many patients because of the retrospective nature of

Figure 3 Transverse sonogram of the bladder 10 weeks after left Leadbetter-Politano reimplantation (with psoas hitch and left ureteral plication) for left primary megaureter in a 20 month old girl. A $18 \mathrm{~mm} \times 27 \mathrm{~mm}$ echogenic mural nodule is seen along the left lateral and posterior bladder wall and trigone (arrows), which at biopsy was found to be a granuloma. The mass gradually decreased in size over the following 2 years.

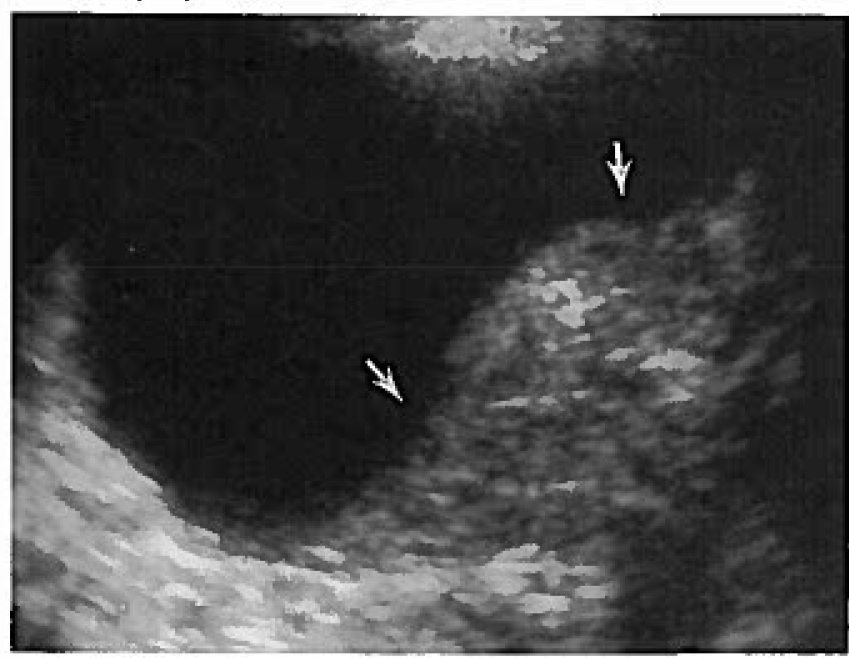




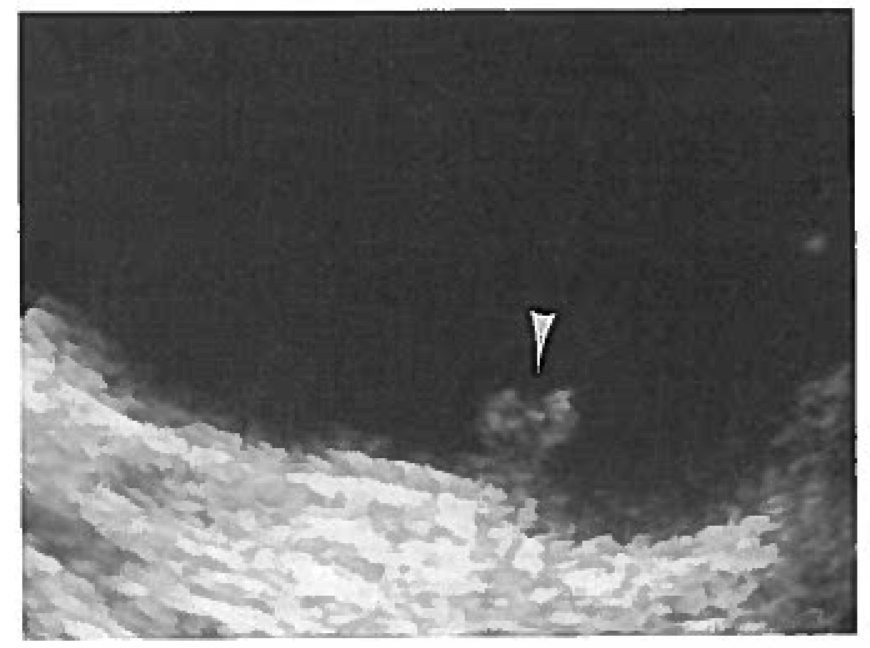

Figure 4 Right parasagittal sonogram of the bladder 3 months after uncomplicated left cross-trigonal reimplantation for left lower pole reflux and left upper pole ureterocele in a 4 year old girl. A polypoid echogenic nodule (arrowhead) is seen on the right side of the trigone, projecting into the bladder lumen.

the study, the nodules clearly decreased in size on follow-up examinations in 10 of 18 patients with multiple post-reimplantation sonograms, disappearing entirely in five subjects.

In 10 of the 14 patients with unilateral reimplantation who had an echogenic nodule visible sonographically, the nodule was on the same side of the trigone as the expected location of the reimplanted ureteral orifice (i.e., contralateral to the side of the reimplanted ureter in the cross-trigonal reimplantation and ipsilateral in the LP reimplantation). In two patients, the nodule was on the opposite side of the trigone from the reimplanted orifice, and in two patients the location of the nodule could not be determined from the images available. Bilateral echogenic nodules were present in three patients who had bilateral cross-trigonal reimplantation (Fig. 5), whereas in six other patients unilateral nodules were visible after bilateral reimplantation.

Seven patients with FBWT on sonography underwent cystoscopy. In one patient a "mass" was seen at the reimplanted orifice, corresponding to an $18 \mathrm{~mm} \times$ $27 \mathrm{~mm}$ nodule first demonstrated sonographically 33 days after reimplantation (Fig. 5). The mass was biopsied and was found to be a granuloma. On follow-up sonography, the mass gradually decreased in size, with only a tiny residual nodule measuring $3 \mathrm{~mm}$ in diameter remaining 2 years later. In another patient, a portion of a mural nodule became calcified (visible at sonography 109 days after reimplantation) and formed an adherent calculus on the trigone that was removed at cystoscopy (Fig. 6). Two patients with an echogenic nodule on the trigone at sonography (55 and 529 days post-reimplantation, respectively) had scarring adjacent to the reimplanted orifice. One patient had "severe

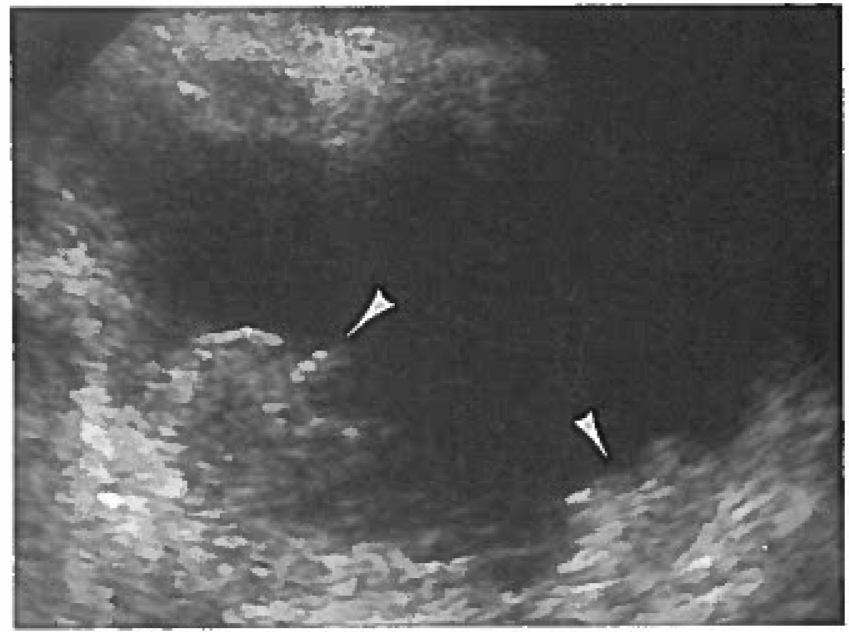

Figure 5 Transverse sonogram of the bladder 3 weeks after uncomplicated bilateral cross-trigonal reimplantation for bilateral reflux in a 7 year old boy. Echogenic nodules are on the trigone bilaterally (arrowheads).

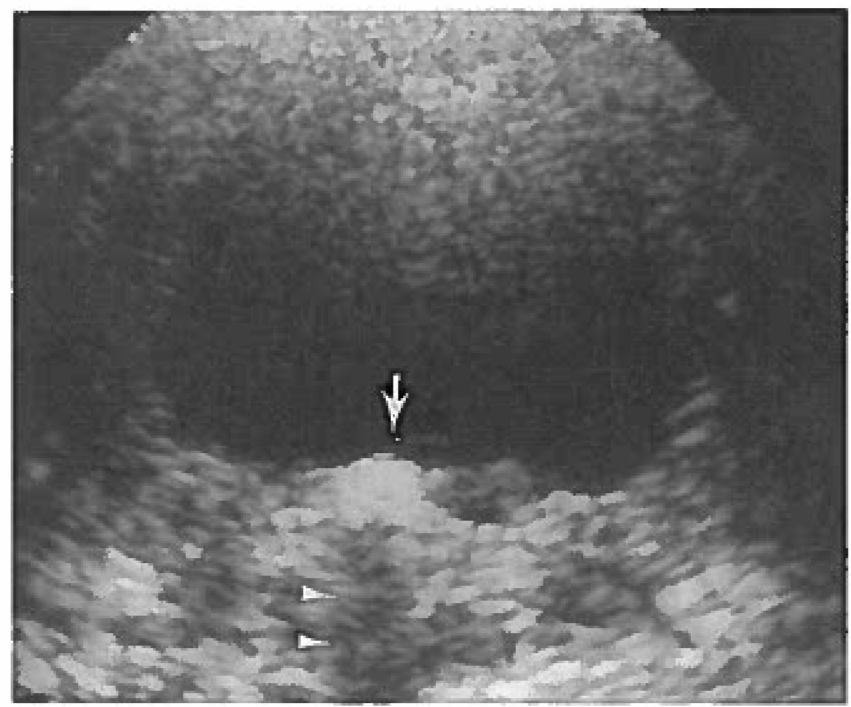

Figure 6 Transverse sonogram of the bladder 3.5 months after right cross-trigonal reimplantation for reflux in a 6 year old girl. An echogenic nodule on the trigone became calcified (arrow) and demonstrated posterior shadowing (arrowheads) on sonography. The calculus was removed at cystoscopy. The child's postoperative course was otherwise uncomplicated.

trabeculation and cellule formation" surrounding the orifice, corresponding to fusiform thickening on sonography 323 days after reimplantation. No abnormality was noted on cystoscopy in one patient with unilateral fusiform thickening of the trigone 97 days after reimplantation.

\section{DISCUSSION}

Focal bladder wall thickening can be seen on sonography after ureteral reimplantation and was visible in $32(54.2 \%)$ of the patients in the present study. Al- 
though the course of the submucosal ureter and the location of the neoureteral orifice are different after cross-trigonal and LP reimplantations (Fig. 1), the changes in the sonographic appearance of the bladder wall are otherwise the same postoperatively for both procedures.

Focal fusiform thickening of the posterior bladder wall and trigone along the expected course of the submucosal tunnel (Fig. 2) can be seen without the ureter itself being dilated. Because the nondilated submucosal ureter may not be visible routinely in unoperated children, ${ }^{7}$ the thickening seen after reimplantation presumably is related to thickening of the wall of the submucosal ureter and the surrounding tissues of the bladder wall.

In the immediate postoperative period, thickening of the bladder wall undoubtedly develops in all patients as a consequence of edema, although the severity of the associated sonographically visible changes and the rapidity with which these changes recede vary. In some patients, the sonographic abnormality rapidly decreased in severity or resolved over a period of months, whereas in other patients the thickening gradually diminished over several years. Edema probably was the cause of the sonographic abnormalities in the patients in whom the thickening rapidly decreased in severity or resolved. On the other hand, fibrosis and granuloma formation are more likely explanations for changes persisting longer than 6 months or more after operation. However, FBWT shown at cystoscopy to be related to scarring or granuloma formation also can change in appearance on sonography over time, although probably more gradually. It is not possible to predict, solely on the basis of sonographic appearance, in which patients the changes are secondary to postoperative edema rather than fibrosis or granuloma formation, nor is it possible to predict in which patients the changes are likely to resolve.

A discrete mural, echogenic nodule was seen on the trigone at or near the expected site of the neoureteral orifice in 24 patients (40.7\%) (Figs. 3 to 5). Previous authors have suggested that the nodule might represent redundancy of tissue at the orifice of the reimplanted ureter. ${ }^{6}$ That the thickening can disappear over time in some patients argues against its representing the neoureteral orifice itself. In the present study, one such nodule was shown to represent a granuloma on biopsy (Fig. 3) and was believed by the surgeon to be related to an exposed Vicryl suture. A second nodule, which became calcified, might also have formed in relation to exposed Vicryl suture material and was removed at cystoscopy (Fig. 6). Adherent clot or a focal inflammatory process at the reimplanted orifice might also conceivably produce a similar appearance.

The sonographic appearance of the echogenic nodule after reimplantation is not specific diagnostically. An identical appearance could be produced by a collapsed ureterocele, focal cystitis, clot, or polyp. The clinical history of reimplantation and the proximity of the nodule to the expected location of the neoureteral orifice are helpful in suggesting the diagnosis.

\section{REFERENCES}

1. Lebowitz RL: Ureter. In Postoperative Pediatric Uroradiology. New York, Appleton-Century-Crofts, 1981, p 15

2. Hendren WH: Ureteral reimplantation in children. J Pediatr Surg 3:649, 1968

3. Cohen S]: Cohen technique of ureteroneocystostomy. In Eckstein HB, Hohenfellner R, Williams DI, eds: Surgical Pediatric Urology. Philadelphia, WB Saunders, 1976, p 269

4. Glassberg KI, Laugani G, Wasnick RJ, et al: Transverse ureteral advancement technique of ureteroneocystostomy (Cohen reimplant) and a modification for difficult cases. J Urol 143:304, 1985

5. Politano VA, Leadbetter WF: An operative technique for the correction of vesicoureteral reflux. J Urol 79:932, 1958

6. Mezzacoppa PM, Price AP, Kassner EG, et al: Cohen ureteral reimplantation: Sonographic appearance. $\mathbf{R a -}$ diology 165:851, 1987

7. Marchal GJ, Baert AL, Eeckels R, et al: Sonographic evaluation of the normal ureteral submucosal tunnel in infancy and childhood. Pediatr Radiol 13:125, 1983 